\title{
Optimization of CHR Propagation Rules
}

\author{
Peter Van Weert* \\ Department of Computer Science, K.U.Leuven, Belgium \\ Peter.VanWeert@cs.kuleuven.be
}

\begin{abstract}
Constraint Handling Rules (CHR) is an elegant, high-level programming language based on multi-headed, forward chaining rules. To ensure CHR propagation rules are applied at most once with the same combination of constraints, CHR implementations maintain a socalled propagation history. The performance impact of this history can be significant. We introduce several optimizations that, for the majority of CHR rules, eliminate this overhead. We formally prove their correctness, and evaluate their implementation in two state-of-the-art CHR systems.
\end{abstract}

\section{Introduction}

Constraint Handling Rules (CHR) [1,2] is a high-level committed-choice CLP language, based on multi-headed, guarded multiset rewrite rules. Originally designed for the declarative specification of constraint solvers, it is increasingly used for general purposes, in a wide range of applications. Efficient implementations exist for several host languages, including Prolog [3], Haskell, and Java [4].

An important, distinguishing feature of CHR are propagation rules. Unlike most rewrite rules, propagation rules do not remove the constraints matched by their head. To avoid trivial non-termination, each CHR rule is therefore applied at most once with the same combination of constraints. This requirement stems from the formal study of properties such as termination and confluence [1], and is reflected in most current CHR implementations.

To prevent reapplication, a CHR runtime system maintains a so-called propagation history, containing a tuple for each constraint combination that fired a rule. Efficiently implementing a propagation history is challenging. Even with the implementation techniques proposed in e.g. [5-7], maintaining a propagation history remains expensive. Our empirical observations reveal that the history often has a significant impact on both space and time performance. Existing literature on CHR compilation nevertheless pays only scant attention to historyrelated optimizations. This paper resolves this discrepancy by introducing several novel approaches to resolve history-related performance issues. We show that, for almost all CHR rules, the propagation history can be eliminated completely. We either use innovative, alternate techniques to prevent rule reapplication, or prove that reapplication has no observable effect. Experimental results confirm the relevance and effectiveness of our optimizations.

\footnotetext{
* Research Assistant of the Research Foundation-Flanders (FWO-Vlaanderen).
} 
Overview Section 3 discusses non-reactive CHR rules-rules that are not reconsidered when built-in constraints are added - and shows that their history can always be eliminated without affecting the program's operational semantics. More precisely, we prove that reapplication of non-reactive rules is either impossible, or that it can be prevented using a novel, more efficient technique.

Section 4 introduces the notion of idempotence. We prove that reapplying idempotent rules has no observable effect, and thus that their history can be eliminated as well, even if the rule is reactive. Together, the optimizations of Sections 3 and 4 cover the majority of the rules found in existing CHR programs.

We implemented the proposed optimizations in two state-of-the-art CHR implementations. Section 5 reports on the significant performance gains obtained. Section 6, finally, reviews some related work and concludes.

For self-containedness, we first briefly review CHR's syntax and operational semantics in Section 2. Gentler introductions are found for instance in $[1,5,6]$.

\section{Preliminaries}

\subsection{CHR Syntax}

CHR is embedded in a host language $\mathcal{H}$. A constraint type $c / n$ is denoted by a functor/arity pair; constraints $c\left(x_{1}, \ldots, x_{n}\right)$ are atoms constructed from these symbols. Their arguments $x_{i}$ are instances of data types offered by $\mathcal{H}$. Many CHR systems support type and mode declarations for constraint arguments.

There are two classes of constraints: built-in constraints, solved by an underlying constraint solver of the host language $\mathcal{H}$, and $C H R$ constraints, handled by a CHR program. A CHR program $\mathcal{P}$, also called a $C H R$ handler, is a sequence of CHR rules. The generic syntactic form of a CHR rule is:

$$
\rho @ H_{k} \backslash H_{r} \Leftrightarrow G \mid B
$$

The rule's unique name $\rho$ is optional; if omitted a name is assigned implicitly. The head consists of two conjunctions of CHR constraints, $H_{k}$ and $H_{r}$. Their conjuncts are called occurrences (kept and removed occurrences resp.). If $H_{k}$ is empty, the rule is a simplification rule. If $H_{r}$ is empty, it is a propagation rule, and ' $\Rightarrow$ ' is used instead of ' $\Leftrightarrow$ '. If both are non-empty, the rule is a simpagation rule. The guard $G$ is a conjunction of built-in constraints, the body $B$ a conjunction of CHR and built-in constraints. A trivial guard 'true |' may be omitted.

Example 1. Fig. 1 shows a classic CHR handler, called LEQ. It defines a single CHR constraint, a less-than-or-equal constraint, using four CHR rules. All three kinds of rules are present. All constraint arguments are logical variables.

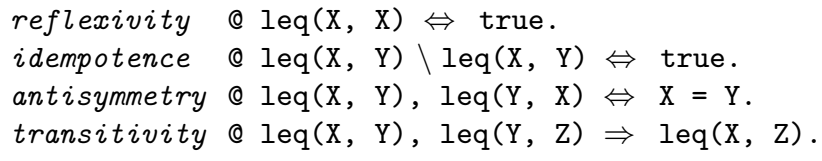

Fig. 1. LEQ, a CHR program for the less-than-or-equal constraint. 
The handler uses a built-in equality constraint $=/ 2$ (e.g. Prolog's built-in unification). The first two rules remove redundant constraints. The antisymmetry rule replaces the CHR constraints matched by its head with a built-in equality constraint. The transitivity propagation rule adds implied CHR constraints.

Head Normal Form In the Head Normal Form of a CHR program $\mathcal{P}$, denoted $\operatorname{HNF}(\mathcal{P})$, variables occur at most once in a rule's head. For instance in $\mathrm{HNF}(\mathrm{LEQ})$, the normalized form of the transitivity rule from Fig. 1 is:

transitivity @ $\operatorname{leq}(\mathrm{X}, \mathrm{Y}), \operatorname{leq}\left(\mathrm{Y}_{1}, \mathrm{Z}\right) \Rightarrow \mathrm{Y}=\mathrm{Y}_{1} \mid \operatorname{leq}(\mathrm{X}, \mathrm{Z})$.

\subsection{CHR's Refined Operational Semantics}

The behavior of most current CHR implementations is captured formally by the refined operational semantics [8], commonly denoted as $\omega_{r}$. The $\omega_{r}$ semantics is formulated as a state transition system, in which transition rules define the relation between subsequent execution states. The version presented here follows $[5,6]$, which is a slight refinement of the original specification [8].

Notation Sets, multisets and sequences (ordered multisets) are defined as usual. We use $S[i]$ to denote the $i$ 'th element of a sequence $S,++$ for sequence concatenation, and $[e \mid S]$ to denote $[e]++S$. The disjoint union of sets is defined as: $\forall X, Y, Z: X=Y \sqcup Z \leftrightarrow X=Y \cup Z \wedge Y \cap Z=\emptyset$. For a logical expression $X$ and a set $V$ of variables, vars $(X)$ denotes the set of free variables, and constraint projection is defined as $\pi_{V}(X) \leftrightarrow \exists v_{1}, \ldots, v_{n}: X$ with $\left\{v_{1}, \ldots, v_{n}\right\}=\operatorname{vars}(X) \backslash V$.

Execution States An execution state of $\omega_{r}$ is a tuple $\langle\mathbb{A}, \mathbb{S}, \mathbb{B}, \mathbb{T}\rangle_{n}$. The role of the execution stack $\mathbb{A}$ is explained below. The $\omega_{r}$ semantics is multiset-based. To distinguish between otherwise identical constraints, the $C H R$ constraint store $\mathbb{S}$ is a set of identified CHR constraints, denoted $c \# i$, where each CHR constraint $c$ is associated with a unique integer number $i$, called a constraint identifier. The projection operators $\operatorname{CHR}(c \# i)=c$ and $\operatorname{ID}(c \# i)=i$ are extended to sequences and sets in the obvious manner. The integer $n$ represents the next available constraint identifier. The built-in constraint store $\mathbb{B}$ is a conjunction containing all built-in constraints passed to the built-in solver. Their meaning is determined by the built-in constraint theory $\mathcal{D}_{\mathcal{H}}$ (see e.g. [6] for a rigorous definition of $\mathcal{D}_{\mathcal{H}}$ ). The propagation history $\mathbb{T}$, finally, is a set of tuples, each recording a sequence of identifiers of CHR constraints that fired a rule, and the name of that rule.

Transition Rules Fig. 2 lists the transition rules of $\omega_{r}$. Execution proceeds by exhaustively applying these transitions, starting from an initial execution state $\langle Q, \emptyset \text {, true, } \emptyset\rangle_{1}$. The constraint sequence $Q$ is called the initial query $Q$.

CHR constraints are assigned unique identifiers and added to $\mathbb{S}$ in Activate transitions. The execution stack $\mathbb{A}$ is a sequence used to treat constraints as procedure calls. The top-most element of $\mathbb{A}$ is called the active constraint. When active, a CHR constraint performs a search for applicable rules. The $\omega_{r}$ semantics specifies that occurrences in a handler are tried in a top-down, right-to-left order. To realize this order in $\omega_{r}$, identified constraints on the execution stack 
1. Solve $\langle[b \mid \mathbb{A}], \mathbb{S}, \mathbb{B}, \mathbb{T}\rangle_{n} \longmapsto_{\mathcal{P}}\langle S++\mathbb{A}, \mathbb{S}, b \wedge \mathbb{B}, \mathbb{T}\rangle_{n}$ if $b$ is a built-in constraint. For the set of reactivated constraints $S \subseteq \mathbb{S}$, the following bounds hold: lower bound: $\forall H \subseteq \mathbb{S}$ : $(\exists K, R: H=K++R \wedge \exists \rho \in \mathcal{P}: \neg \operatorname{appl}(\rho, K, R, \mathbb{B}) \wedge \operatorname{appl}(\rho, K, R, b \wedge \mathbb{B})) \rightarrow(S \cap H \neq \emptyset)$ and upper bound: $\forall c \in S: \operatorname{vars}(c) \not \subset \operatorname{fixed}(\mathbb{B})$.

2. Activate $\langle[c \mid \mathbb{A}], \mathbb{S}, \mathbb{B}, \mathbb{T}\rangle_{n} \longmapsto_{\mathcal{P}}\langle[c \# n: 1 \mid \mathbb{A}],\{c \# n\} \sqcup \mathbb{S}, \mathbb{B}, \mathbb{T}\rangle_{n+1}$ if $c$ is a CHR constraint (which has not yet been active or stored in $\mathbb{S}$ ).

3. Reactivate $\langle[c \# i \mid \mathbb{A}], \mathbb{S}, \mathbb{B}, \mathbb{T}\rangle_{n} \succ_{\mathcal{P}}\langle[c \# i: 1 \mid \mathbb{A}], \mathbb{S}, \mathbb{B}, \mathbb{T}\rangle_{n}$ if $c$ is a CHR constraint (re-added to $\mathbb{A}$ by a Solve transition but not yet active).

4. Simplify $\langle[c \# i: j \mid \mathbb{A}], \mathbb{S}, \mathbb{B}, \mathbb{T}\rangle_{n} \succ_{\mathcal{P}}\left\langle B++\mathbb{A}, K \sqcup S, \theta \wedge \mathbb{B}, \mathbb{T}^{\prime}\right\rangle_{n}$ with $\mathbb{S}=\{c \# i\} \sqcup$ $K \sqcup R_{1} \sqcup R_{2} \sqcup S$, if the $j$-th occurrence of $c$ in $\mathcal{P}$ occurs in rule $\rho$, and $\theta$ is a matching substitution such that $\operatorname{apply}\left(\rho, K, R_{1}++[c \# i]++R_{2}, \mathbb{B}, \theta\right)=B$.

Let $t=\left(\rho, \operatorname{ID}\left(K++R_{1}\right)++[i]++\operatorname{ID}\left(R_{2}\right)\right)$, then $t \notin \mathbb{T}$ and $\mathbb{T}^{\prime}=\mathbb{T} \cup\{t\}$.

5. Propagate $\langle[c \# i: j \mid \mathbb{A}], \mathbb{S}, \mathbb{B}, \mathbb{T}\rangle_{n} \longmapsto_{\mathcal{P}}\left\langle B+[c \# i: j \mid \mathbb{A}], \mathbb{S} \backslash R, \theta \wedge \mathbb{B}, \mathbb{T}^{\prime}\right\rangle_{n}$ with $\mathbb{S}=$ $\{c \# i\} \sqcup K_{1} \sqcup K_{2} \sqcup R \sqcup S$, if the $j$-th occurrence of $c$ in $\mathcal{P}$ occurs in rule $\rho$, and $\theta$ is a matching substitution such that $\operatorname{apply}\left(\rho, K_{1}+[c \# i]++K_{2}, R, \mathbb{B}, \theta\right)=B$.

Let $t=\left(\rho, \operatorname{ID}\left(K_{1}\right)+[i]++\operatorname{ID}\left(K_{2}++R\right)\right)$, then $t \notin \mathbb{T}$ and $\mathbb{T}^{\prime}=\mathbb{T} \cup\{t\}$.

6. Drop $\langle[c \# i: j \mid \mathbb{A}], \mathbb{S}, \mathbb{B}, \mathbb{T}\rangle_{n} \longmapsto_{\mathcal{P}}\langle\mathbb{A}, \mathbb{S}, \mathbb{B}, \mathbb{T}\rangle_{n}$ if $c$ has no $j$-th occurrence in $\mathcal{P}$.

7. Default $\langle[c \# i: j \mid \mathbb{A}], \mathbb{S}, \mathbb{B}, \mathbb{T}\rangle_{n} \longmapsto_{\mathcal{P}}\langle[c \# i: j+1 \mid \mathbb{A}], \mathbb{S}, \mathbb{B}, \mathbb{T}\rangle_{n}$ if the current state cannot fire any other transition.

Fig. 2. The transition rules of the refined operational semantics $\omega_{r}$.

are occurrenced. If an occurrenced identified CHR constraint $c \# i: j$ is active, only matches with the $j$ 'th occurrence of $c$ 's constraint type are considered. Interleaving a sequence of Default transitions, all applicable rules are thus fired in Propagate and Simplify transitions. A rule is applicable if the store contains matching partner constraints for all remaining occurrences in its head. Formally:

Definition 1. Given a conjunction of built-in constraints $\mathbb{B}$, a rule $\rho$ is applicable with sequences of identified $C H R$ constraints $K$ and $R$, denoted appl $(\rho, K, R, \mathbb{B})$, iff a matching substitution $\theta$ exists for which apply $(\rho, K, R, \mathbb{B}, \theta)$ is defined. The latter partial function is defined as apply $(\rho, K, R, \mathbb{B}, \theta)=B$ iff $K \cap R=\emptyset$ and, renamed apart, $\rho$ is of form " $\rho @ H_{k} \backslash H_{r} \Leftrightarrow G \mid B$ " ( $H_{k}$ or $H_{r}$ may be empty) with $\operatorname{CHR}(K)=\theta\left(H_{k}\right), \operatorname{CHR}(R)=\theta\left(H_{r}\right)$, and $\mathcal{D}_{\mathcal{H}} \models \mathbb{B} \rightarrow \pi_{\text {vars }(\mathbb{B})}(\theta \wedge G)$.

If the top-most element of $\mathbb{A}$ is a built-in constraint, this constraint is passed to the built-in solver in a Solve transition. As this may affect the entailment of guards, all CHR constraints for which additional rules might have become applicable have to be put back on the execution stack. These then cause Reactivate transitions to reinitiate searches for applicable rules. Constraints with fixed arguments are not reactivated, as no additional guards can become entailed.

Definition 2. A variable $v$ is fixed by constraint conjunction $B$, or $v \in f i x e d(B)$, iff $\mathcal{D}_{\mathcal{H}} \models \forall \theta\left(\left(\pi_{\{v\}}(B) \wedge \pi_{\{\theta(v)\}}(\theta(B))\right) \rightarrow v=\theta(v)\right)$ for any variable renaming $\theta$.

When a rule fires, its body is executed. By putting the body on the activation stack, the different conjuncts of the body are activated (for CHR constraints) or 


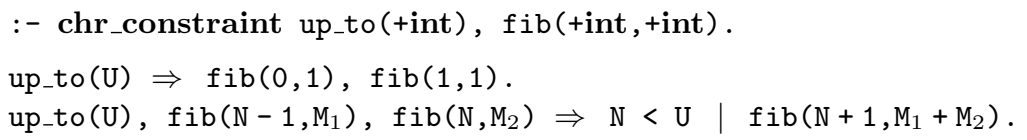

Fig. 3. This handler, referred to as FIBBO, performs a bottom-up computation of all Fibonacci numbers up to a given number. All constraint arguments are fixed integers.

solved (for built-in constraints) in a left-to-right order. Control only returns to the original active constraint after the body is completely executed.

Derivations For a CHR operational semantics $\omega$, an $\omega$-derivation $D$ is a (possibly infinite) sequence of $\omega_{r}$ states, with $D[1]$ an initial execution state for some query $Q$, and $D[i] \longmapsto_{\mathcal{P}} D[i+1]$ valid $\omega$ transitions. We use the notational abbreviation $\sigma_{1} \longmapsto_{\mathcal{P}}^{\star} \sigma_{n}$ to denote a finite derivation $\left[\sigma_{1}, \ldots, \sigma_{n}\right]$.

\section{Non-reactive Propagation Rules}

Section 3.1 introduces non-reactive CHR rules, rules that are never matched by a reactivated constraint, and illustrates that a substantial portion of CHR rules is non-reactive. In Section 3.2, we prove that the history of certain non-reactive propagation rules can be eliminated, as CHR's operational semantics ensures these rules are never matched by the same constraint combination. For the remaining non-reactive rules, we introduce an innovative, more efficient technique to prevent rule reapplication in Section 3.3, and prove its soundness.

\subsection{Introduction: From Fixed to Non-reactive CHR}

Non-reactive CHR constraints are never reactivated when built-in constraints are added. Formally:

Definition 3. A CHR constraint type $c / n$ is non-reactive in a program $\mathcal{P}$ under a refined operational semantics $\omega_{r}^{\star}\left(\omega_{r}\right.$ or any of its refinements: see further) iff for any Solve transitions of the form $\langle[b \mid \mathbb{A}], \mathbb{S}, \mathbb{B}, \mathbb{T}\rangle_{n} \longmapsto_{\mathcal{P}}\langle S++\mathbb{A}, \mathbb{S}, b \wedge \mathbb{B}, \mathbb{T}\rangle_{n}$ in any $\omega_{r}^{\star}$-derivation $D$ the set of reactivated constraints $S \subseteq \mathbb{S}$ does not contain constraints of type $c / n$. A rule $\rho \in \mathcal{P}$ is non-reactive iff all constraint types that occur in its head are non-reactive in $\mathcal{P}$.

The simplest instances are so-called fixed constraints. A CHR constraint type $c / n$ is fixed iff $\operatorname{var} s(c) \subseteq$ fixed $(\emptyset)$ (see Definition 2) for all constraints $c$ of this type. Clearly, if all constraint arguments are fixed, no additional rule becomes applicable when adding built-in constraints. Which CHR constraints are fixed is derived from their mode declarations, or using static groundness analysis [9].

Example 2. The FIBBo handler depicted in Fig. 3, performs a bottom-up computation of all Fibonacci numbers up to a given number. The constraint declarations ${ }^{1}$ specify that all arguments are fixed instances of the host language's int type (the '+' mode declaration indicates a constraint's argument is fixed).

\footnotetext{
${ }^{1}$ The syntax is inspired by that of the K.U.Leuven CHR system $[3,6]$.
} 


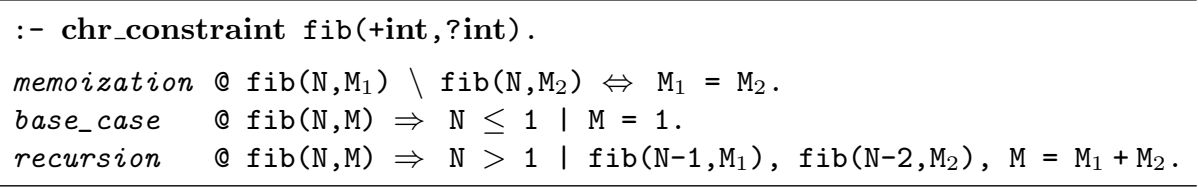

Fig. 4. A CHR handler that computes Fibonacci numbers using a top-down computation strategy with memoization.

Under $\omega_{r}$, a CHR constraint type is non-reactive iff it is fixed. The following example though shows why the class of non-reactive constraints should be larger:

Example 3. Fig. 4 contains an alternative Fibonacci handler, this time using a top-down computation strategy with memoization. The $\mathrm{fib} / 2$ constraint is not fixed, and is typically called with a free (logical) variable as second argumenthence also the '?' mode declaration. Reactivating $\mathrm{fib} / 2$ constraints is nevertheless pointless, as there are no guards constraining its second argument. Additional built-in constraints therefore never result in additional applicable rules.

All theoretical results in this section apply to non-reactive rules only. Under $\omega_{r}$, however, constraints such as $\mathrm{fib} / 2$ are not non-reactive. As using unbound, unguarded arguments to retrieve results is very common in CHR, a minor refinement $\omega_{r}$ is required to increase the practical relevance of our results.

In general, CHR constraints should only be reactivated if extra built-in constraints may cause more guards to become entailed. We therefore reintroduce the concept of anti-monotonicity [7, 10]:

Definition 4. A conjunction of built-in constraints $B$ is anti-monotone in a set of variables $V$ iff $\forall B_{1}, B_{2}\left(\left(\pi_{\text {vars }(B) \backslash V}\left(B_{1} \wedge B_{2}\right) \leftrightarrow \pi_{\text {vars }(B) \backslash V}\left(B_{1}\right)\right)\right.$

$$
\left.\rightarrow\left(\left(\mathcal{D}_{\mathcal{H}} \not \models B_{1} \rightarrow B\right) \rightarrow\left(\mathcal{D}_{\mathcal{H}} \not \models B_{1} \wedge B_{2} \rightarrow B\right)\right)\right)
$$

Definition 5. A CHR program $\mathcal{P}$ is anti-monotone in the $i$ 'th argument of a CHR constraint type $c / n$, if and only if for every occurrence $c\left(x_{1}, \ldots, x_{i}, \ldots, x_{n}\right)$ in $\operatorname{HNF}(\mathcal{P})$, the guard of the corresponding rule is anti-monotone in $\left\{x_{i}\right\}$.

Any CHR program is anti-monotone in both fixed and unguarded constraint arguments. Moreover, several typical built-ins are anti-monotone in their arguments. In Prolog, for instance, $\operatorname{var}(\mathrm{X})$ is anti-monotone in $\{\mathrm{X}\}$. Using antimonotonicity, we now define $\omega_{r}^{\prime}$, a slight refinement of $\omega_{r}^{2}$ :

Definition 6. Let delay_vars $s_{\mathcal{P}}(c)$ denote the set of variables in which $\mathcal{P}$ is not anti-monotone that occur in an (identified) CHR constraint $c$. Then $\omega_{r}^{\prime}$ is obtained from $\omega_{r}$ by replacing the upper bound on the set of reactivated constraints $S$ in its Solve transition with " $\forall c \in S:$ delay_vars $s_{\mathcal{P}}(c) \not \subset$ fixed $(\mathbb{B})$ ".

Most rules in general-purpose CHR programs are non-reactive under $\omega_{r}^{\prime}$. Several CHR systems, including the K.U.Leuven CHR and JCHR systems [3, 4], implement $\omega_{r}^{\prime}$. Doing so, may already improve performance considerably (see [10]). In the following two subsections, we prove that for non-reactive CHR rules the expensive maintenance of a propagation history can always be avoided.

\footnotetext{
$\overline{{ }^{2} \text { We refer to }}\left[7\right.$, Appendix A] for a formal proof that $\omega_{r}^{\prime}$ is indeed an instance of $\omega_{r}$.
} 


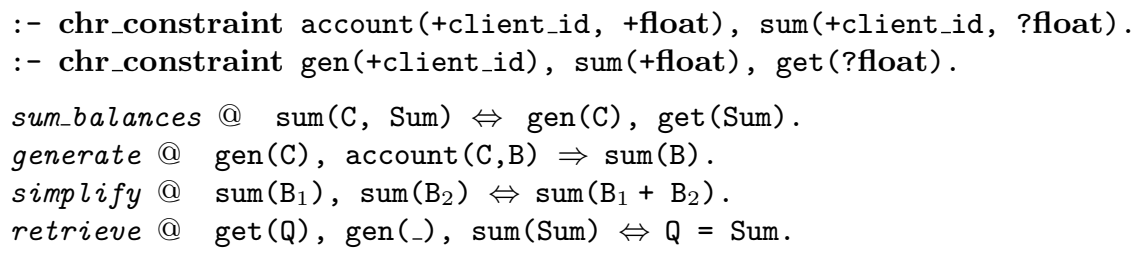

Fig. 5. CHR rules computing the sum of the account balances of a given client. These rules may be part of some larger CHR handler modeling a banking application.

\subsection{Propagation History Elimination}

Because non-reactive CHR constraints are only active once, non-reactive propagation rules often do not require a history:

Example 4. The sum/2 constraint in Fig. 5 computes the sum of a client's account balances using a common CHR programming idiom to compute aggregates: a (typically non-reactive) propagation rule generates a number of constraints, from which, after simplification to a single constraint, the result can be retrieved.

When the active gen/1 constraint considers the generate rule, it iterates over candidate account $/ 2$ partner constraints. Assuming this iteration does not contain duplicates (a property formalized shortly in Definition 8), the generate rule never fires with the same constraint combination under $\omega_{r}$, even if no propagation history is maintained. Indeed, the generate rule only adds sum/1 constraints, which, as there is no get/1 constraint yet in the store (the body of the sum_balances rule is executed from left to right), only fire the simplify rule.

The history, however, is not superfluous for all non-reactive CHR rules, as shown by the following example:

Example 5. Reconsider the FIBBO handler of Fig. 3. If an up_to(U) constraint is told, the first rule propagates two $\mathrm{fib} / 2$ constraints. After this, the second rule propagates all required $f i b / 2$ constraints, each time with a fib/2 constraint as the active constraint. Next, control returns to the up_to(U) constraint, and advances to its second occurrence. Some mechanism is then required to prevent the second (non-reactive) propagation rule to add erroneous $\mathrm{fib} / 2$ constraints.

So, non-reactive propagation rules can match the same constraint combination more than once. This occurs if one or more partner constraints for an active constraint in rule $\rho$ were added by firing $\rho$ or some earlier rule, whilst the same constraint was already active. We say these partner constraints observe the corresponding occurrence of the active constraint in $\rho$ (cf. also [9]). Formally:

Definition 7. Let the $k$ 'th occurrence of a rule $\rho$ 's head be the $j$ 'th occurrence of constraint type $c / n$. Then this occurrence is unobserved under a refined operational semantics $\omega_{r}^{\star}$ iff for all Activate or Default transitions of the form ${ }^{3}$ :

$$
\left\langle\mathbb{A}_{0}, \mathbb{S}, \mathbb{B}, \mathbb{T}\right\rangle_{-} \longmapsto_{\mathcal{P}}\langle[c \# i: j \mid \mathbb{A}], \mathbb{S}, \mathbb{B}, \mathbb{T}\rangle_{-}
$$

\footnotetext{
${ }^{3}$ We use '_' to denote that we are not interested in the identifier counter.
} 
$\left(\mathbb{A}_{0}[1]=c \# i\right.$ or $\left.\mathbb{A}_{0}[1]=c \# i: j-1\right)$ the following holds: $\forall(\rho, I) \in \mathbb{T}: I[k] \neq i$, and similarly for all transition sequences starting with a Propagate transition

$$
\langle\mathbb{A}, \mathbb{S}, \mathbb{B}, \mathbb{T}\rangle_{-} \succ_{\mathcal{P}}\left\langle B++\mathbb{A}, \mathbb{S}^{\prime}, \mathbb{B}^{\prime}, \mathbb{T}^{\prime}\right\rangle_{-} \succ_{\mathcal{P}}^{\star}\left\langle\mathbb{A}, \mathbb{S}^{\prime \prime}, \mathbb{B}^{\prime \prime}, \mathbb{T}^{\prime \prime}\right\rangle_{-}
$$

with $\mathbb{A}[1]=c \# i: j, \forall(\rho, I) \in \mathbb{T}^{\prime \prime} \backslash \mathbb{T}^{\prime}: I[k] \neq i$.

Let $\omega_{r}^{\dagger}$ denote the semantics obtained from $\omega_{r}^{\prime}$ by adding the following condition to its Propagate and Simplification transitions: "If the $j$ 'th occurrence of $c$ is unobserved under $\omega_{r}^{\prime}$, then $\mathbb{T}^{\prime}=\mathbb{T}$ ". Also, to prevent trivial reapplication in a consecutive sequence of Propagate transitions (see e.g. Example 4), propagation in $\omega_{r}^{\dagger}$ is defined to be duplicate-free:

Definition 8 (Duplicate-free Propagation). Propagation in a refined operational semantics $\omega_{r}^{\star}$ is duplicate-free iff for all $\omega_{r}^{\star}$-derivations $D$ of a CHR program $\mathcal{P}$ where the $j^{\prime}$ 'th occurrence of $c$ is kept, the following holds:

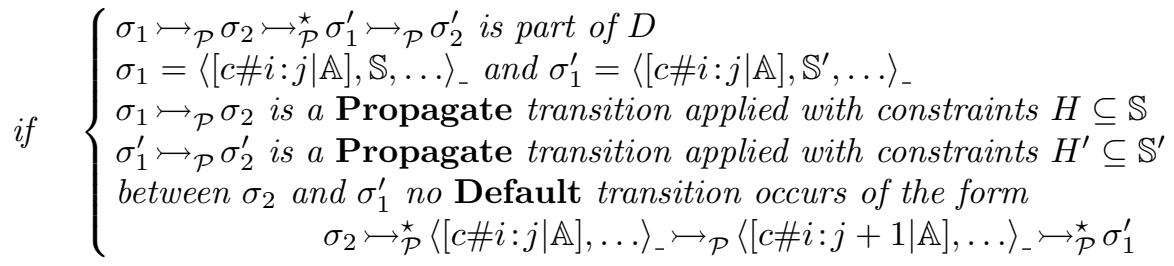
then $H \neq H^{\prime}$.

The following theorem establishes the equivalence of $\omega_{r}^{\dagger}$ and $\omega_{r}^{\prime}$, thus proving the soundness of eliminating the history of unobserved CHR rules:

Theorem 1. Define the mapping function $\alpha^{\dagger}$ as follows:

$$
\alpha^{\dagger}\left(\langle\mathbb{A}, \mathbb{S}, \mathbb{B}, \mathbb{T}\rangle_{n}\right)=\langle\mathbb{A}, \mathbb{S}, \mathbb{B},\{(\rho, I) \in \mathbb{T} \mid \rho \text { is not unobserved }\}\rangle_{n}
$$

If $D$ is an $\omega_{r}^{\prime}$ derivation, then $\alpha^{\dagger}(D)$ is an $\omega_{r}^{\dagger}$ derivation. Conversely, if $D$ is an $\omega_{r}^{\dagger}$ derivation, then there exists an $\omega_{r}^{\prime}$ derivation $D^{\prime}$ such that $\alpha^{\dagger}(D)=D^{\prime}$.

Proof. See [11].

Implementation The main difficulty in the implementation of this optimization is deriving that a rule is unobserved (enforcing duplicate-free propagation is typically straightforward, as shown in Section 3.3). The abstract interpretationbased late storage analysis of [9], which derives a similar observation property, can be adapted for this purpose. The details are beyond the scope of this paper.

\subsection{Optimized Reapplication Avoidance}

Non-reactive CHR rules that are not unobserved, such as the second rule in the FIBBO handler of Example 5, do require some mechanism to prevent reapplication. Moreover, even if a rule is unobserved, this does not mean the compiler's analysis is capable of deriving it. In this section we therefore present a novel, very efficient technique that prevents the reapplication of any non-reactive propagation rule without maintaining a costly propagation history.

The central observation is that, when a non-reactive rule is applied, the active constraint is always more recent than its partner constraints: 
Lemma 1. Let $\mathcal{P}$ be an arbitrary CHR program, with $\rho \in \mathcal{P}$ a non-reactive rule, and $D$ an arbitrary $\omega_{r}^{\prime}$ derivation with this program. Then for each Simplify or Propagate transition in $D$ of the form

$$
\langle[c \# i: j \mid \mathbb{A}], \mathbb{S}, \mathbb{B}, \mathbb{T}\rangle_{n} \mapsto_{\mathcal{P}}\left\langle\mathbb{A}^{\prime}, \mathbb{S}^{\prime}, \mathbb{B}^{\prime}, \mathbb{T} \sqcup\left\{\left(\rho, I_{1}+[i]++I_{2}\right)\right\}\right\rangle_{n}
$$

the following holds: $\forall i^{\prime} \in I_{1} \cup I_{2}: i^{\prime}<i$.

Proof. Assume $i^{\prime}=\max \left(I_{1} \sqcup I_{2}\right)$ with $i^{\prime} \geq i$. By Definition 1 of rule applicability, $i^{\prime} \neq i$, and $\exists c^{\prime} \# i^{\prime} \in \mathbb{S}$. This $c^{\prime} \# i^{\prime}$ partner constraint must have been stored in an Activate transition. Since $i^{\prime}=\max \left(I_{1} \sqcup\{i\} \sqcup I_{2}\right)$, in $D$, this transition came after the Activate transitions of all other partners, including $c \# i$. In other words, all constraints in the matching combination of transition (1) were stored prior to the activation of $c^{\prime} \# i^{\prime}$. Also, in (1), $c \# i$ is back on top of the activation stack. Because $c$ is non-reactive, and thus never put back on top by a Reactivate transition, the later activated $c^{\prime} \# i^{\prime}$ must have been removed from the stack in a Drop transition. This implies that all applicable rules matching $c^{\prime}$ must have fired. As all required constraints were stored (see earlier), this includes the application of $\rho$ in (1). By contradiction, our assumption is false, and $i^{\prime}<i$.

Let $\omega_{r}^{\ddagger}$ denote the semantics obtained from $\omega_{r}^{\prime}$ by replacing the propagation history condition in its Simplify and Propagate transitions with the following:

If $\rho$ is non-reactive, then $\forall i^{\prime} \in \operatorname{ID}\left(H_{1} \cup H_{2}\right): i^{\prime}<i$ and $\mathbb{T}^{\prime}=\mathbb{T}$. Otherwise, let $t=\left(\rho, \operatorname{ID}\left(H_{1}\right)++[i]++\operatorname{ID}\left(H_{2}\right)\right)$, then $t \notin \mathbb{T}$ and $\mathbb{T}^{\prime}=\mathbb{T} \cup\{t\}$.

Propagation in $\omega_{r}^{\ddagger}$ is again duplicate-free, as defined by Definition 8. Similarly to Theorem 1, the following theorem proves that $\omega_{r}^{\prime}$ and $\omega_{r}^{\ddagger}$ are equivalent:

Theorem 2. Define the mapping function $\alpha^{\ddagger}$ as follows:

$$
\alpha^{\ddagger}\left(\langle\mathbb{A}, \mathbb{S}, \mathbb{B}, \mathbb{T}\rangle_{n}\right)=\langle\mathbb{A}, \mathbb{S}, \mathbb{B},\{(\rho, I) \in \mathbb{T} \mid \rho \text { is a reactive CHR rule }\}\rangle_{n}
$$

If $D$ is an $\omega_{r}^{\prime}$ derivation, then $\alpha^{\ddagger}(D)$ is an $\omega_{r}^{\ddagger}$ derivation. Conversely, if $D$ is an $\omega_{r}^{\ddagger}$ derivation, then there exists an $\omega_{r}^{\prime}$ derivation $D^{\prime}$ such that $\alpha^{\ddagger}(D)=D^{\prime}$.

Proof. See [7] or [11].

Implementation The standard CHR compilation scheme (see e.g. $[5,6])$ generates for each occurrence a nested iteration that looks for matching partner constraints for the active constraint. If a matching combination is found, and the active constraint is not removed, the constraint iterators are suspended and the rule's body is executed. Afterwards, the nested iteration is simply resumed.

Example 6. Fig. 6(a) shows the generated code for the second occurrence of the up_to/1 constraint in Fig. 3. For the query up_to(U), the propagation history for the corresponding rule would require $\mathcal{O}(U)$ space. Because all constraints are non-reactive, however, no propagation history has to be maintained. Simply comparing constraint identifiers suffices.

If all iterators return candidate partner constraints at most once, propagation is guaranteed to be duplicate-free (see Definition 8). Most iterators used by CHR implementations have this property. If not, a temporary history can for instance be maintained whilst the active constraint is considering an occurrence. 


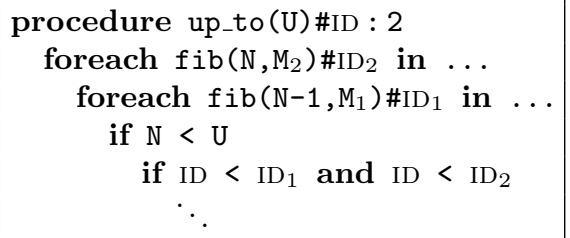

(a) Efficient reapplication avoidance using identifier comparisons

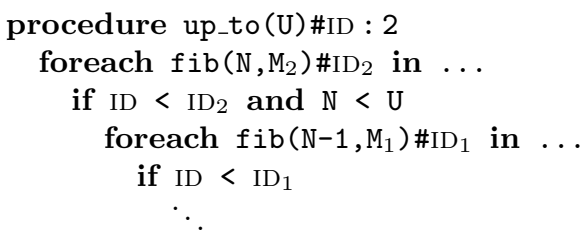

(b) After Loop-invariant Code Motion

Fig. 6. Pseudocode for the second occurrence of the up_to/1 constraint of Fig. 3.

Loop-invariant Code Motion Most CHR compilers perform so-called Loopinvariant Code Motion optimization to check guard entailment as soon as possible (e.g. ' $\mathrm{N}<\mathrm{U}$ ' in Fig. 6(b)). Contrary to a propagation history check, identifier comparisons enable additional code motion, as illustrated in Fig. 6(b). This may prune the search space of candidate partner constraints considerably.

Note furthermore that Lemma 1 does not only apply to propagation rules, but also to simplification and simpagation rules. Whilst maintaining a history for non-propagation rules is pointless, comparing partner constraint identifiers in outer loops is not, as they may avoid redundant iterations of nested loops.

\section{Idempotence}

Constraints in CHR handlers that specify traditional constraint solvers, such as the leq/2 constraint of Example 1, typically range over unbound variables, and are thus highly reactive. Without a history, constraint reactivations may cause reactive propagation rules to fire multiple times with the same combination. For constraint solvers, however, such additional rule applications typically have no effect, as they only add redundant constraints that are immediately removed. For such rules, the propagation history may be eliminated as well.

Example 7. Suppose the reactive transitivity propagation rule of Fig. 1 is allowed to fire a second time with the same constraint combination matching its head, thus adding a leq $(\mathrm{X}, \mathrm{Z})$ constraint for the second time. If the earlier told duplicate is still in the store, this redundant $\operatorname{leq}(\mathrm{X}, \mathrm{Z})$ constraint is immediately removed by the idempotence rule. Otherwise, the former duplicate must have been removed by either the reflexivity or the antisymmetry rule. It is easy to see that in this case $\mathrm{X}=\mathrm{Z}$, and thus that the new, redundant leq $(\mathrm{X}, \mathrm{Z})$ constraint is again removed immediately by the reflexivity rule.

We say the leq/2 constraint of the above example is idempotent. With live $(\mathbb{T}, \mathbb{S})=\{(\rho, I) \in \mathbb{T} \mid I \subseteq \mathrm{ID}(\mathbb{S})\}$, idempotence is defined formally as:

Definition 9. A CHR constraint type $c / n$ is idempotent in a CHR program $\mathcal{P}$ under a refined semantics $\omega_{r}^{\star}$ iff for any state $\sigma=\langle[c \mid \mathbb{A}], \mathbb{S}, \mathbb{B}, \mathbb{T}\rangle_{n}$ in a $\omega_{r}^{\star}$ derivation $D$ with c a CHR constraint, the following holds: if earlier in $D$ a state $\left\langle\left[c^{\prime} \mid \mathbb{A}^{\prime}\right], \mathbb{S}^{\prime}, \mathbb{B}^{\prime}, \mathbb{T}^{\prime}\right\rangle_{n^{\prime}}$ occurs with $\mathcal{D}_{\mathcal{H}}=\mathbb{B} \rightarrow c=c^{\prime}$, then $\sigma \succ_{\mathcal{P}}^{\star}\left\langle\mathbb{A}, \mathbb{S}^{\prime \prime}, \mathbb{B}^{\prime \prime}, \mathbb{T}^{\prime \prime}\right\rangle_{n^{\prime \prime}}$ with $\mathbb{S}^{\prime \prime}=\mathbb{S}$, live $\left(\mathbb{T}^{\prime \prime}, \mathbb{S}\right)=\operatorname{live}(\mathbb{T}, \mathbb{S})$, and $\mathcal{D}_{\mathcal{H}} \models \pi_{\text {vars }(\mathbb{B}) \cup \operatorname{vars}(D[1])}\left(\mathbb{B}^{\prime \prime}\right) \leftrightarrow \mathbb{B}$. 
In other words, an idempotent constraint $c$ for which a syntactically equal constraint $c^{\prime}$ was told earlier in the same derivation, is removed without making any observable state change. Since ${ }^{\prime} \longrightarrow_{\mathcal{P}}^{\star}$ ' denotes a finite derivation, telling duplicate idempotent CHR constraints also does not affect termination.

We do not consider arbitrary, extra-logical host language statements here, and assume all built-in constraints $b$ are idempotent, that is: $\forall b: \mathcal{D}_{\mathcal{H}} \models b \wedge b \leftrightarrow b$. By adding "If $\mathcal{D}_{\mathcal{H}}=(\mathbb{B} \wedge b) \leftrightarrow \mathbb{B}$, then $S=\emptyset$ " to the Solve transition of $\omega_{r}$ (or any of its refinements from Section 3), we avoid redundant constraint reactivations when idempotent built-in constraints are told. This is correct, as Solve's upper bound on $S$ already specifies that any matching already possible prior to $b$ 's addition may be omitted from $S$. Most CHR systems already implement this optimization. Denote the resulting semantics $\omega_{r}^{\text {idem }}$.

Definition 10. A CHR rule $\rho \in \mathcal{P}$ is idempotent under $\omega_{r}^{\text {idem }}$ iff all $C H R$ constraint types that occur in its body are idempotent in $\mathcal{P}$ under $\omega_{r}^{\text {idem }}$.

We now prove that an idempotent propagation rule may be fired more than once with the same combination of constraints, without affecting a program's operational semantics. Let $\omega_{r}^{\text {idem }}$ denote the semantics obtained by adding the following phrase to the Simplify and Propagate transitions of $\omega_{r}^{\text {idem }}$ :

If the rule $\rho$ is idempotent, then $\mathbb{T}^{\prime}=\mathbb{T}$; otherwise, ... (as before)

Assuming furthermore that propagation for $\omega_{r}^{i d e m^{\prime}}$ is duplicate-free ${ }^{4}$ in the sense of Definition 8, the $\omega_{r}^{\text {idem }}$ semantics is equivalent to $\omega_{r}^{\text {idem }}$. More precisely:

Theorem 3. If $D^{\prime}$ is an $\omega_{r}^{\text {idem' }}$ derivation, then there exists an $\omega_{r}^{\text {idem }}$ derivation $D$ with $D[1]=D^{\prime}[1]$ such that a monotonic function $\alpha$ can be defined from the states in $D$ to states in $D^{\prime}$ for which

- $\alpha(D[1])=D^{\prime}[1]$

- if $\alpha(D[i])=D^{\prime}[k]$ and $\alpha(D[j])=D^{\prime}[l]$ with $i<j$, then $k<l$

- if $\alpha\left(\langle\mathbb{A}, \mathbb{S}, \mathbb{B}, \mathbb{T}\rangle_{n}\right)=\left\langle\mathbb{A}^{\prime}, \mathbb{S}^{\prime}, \mathbb{B}^{\prime}, \mathbb{T}^{\prime}\right\rangle_{n^{\prime}}$, then $\mathcal{D}_{\mathcal{H}} \models \pi_{\text {vars }(\mathbb{B}) \cup \operatorname{vars}(D[1])}\left(\mathbb{B}^{\prime}\right) \leftrightarrow \mathbb{B}$, $\mathbb{A}^{\prime}=\mathbb{A}, \mathbb{S}^{\prime}=\mathbb{S}$, and live $\left(\mathbb{T}^{\prime}, \mathbb{S}\right)=$ live $(\mathbb{T}, \mathbb{S}) \backslash\{(\rho, I) \in \mathbb{T} \mid \rho$ is idempotent $\}$.

Conversely, if $D$ is an $\omega_{r}^{\text {idem }}$ derivation, then an $\omega_{r}^{\text {idem }}$ derivation $D^{\prime}$ exists with $D^{\prime}[1]=D[1]$ for which a function with these same properties can be defined.

Proof Sketch. An $\omega_{r}^{\text {idem }}$ derivation $D^{\prime}$ only differs from the corresponding $\omega_{r}^{\text {idem }}$ derivation $D$ when a Propagate transition fires an idempotent propagation rule $\rho$ using a combination of constraints that fired $\rho$ before. This $\omega_{r}^{\text {idem }}$ transition has form $\sigma_{0}=\langle\mathbb{A}, \mathbb{S}, \mathbb{B}, \mathbb{T}\rangle_{n}{\varliminf_{\mathcal{P}}}\langle B+\mathbb{A}, \mathbb{S}, \mathbb{B}, \mathbb{T}\rangle_{n}=\sigma_{1}$. Because $\rho$ 's body $B$ is idempotent, it follows from Definition 9 that the remainder of $D^{\prime}$ begins with $\sigma_{1} \hookrightarrow_{\mathcal{P}}^{\star} \sigma_{0}^{\prime}=\left\langle\mathbb{A}, \mathbb{S}, \mathbb{B}^{\prime}, \mathbb{T}^{\prime}\right\rangle_{n}$, with $\mathcal{D}_{\mathcal{H}} \models \pi_{\text {vars }(\mathbb{B}) \cup \operatorname{vars}(D[1])}\left(\mathbb{B}^{\prime}\right) \leftrightarrow \mathbb{B}$, and live $\left(\mathbb{T}^{\prime}, \mathbb{S}\right)=$ live $(\mathbb{T}, \mathbb{S})$. Because $\sigma_{0}^{\prime}$ is thus essentially equivalent to $\sigma_{0}$, we simply omit states $\sigma_{1}$ to $\sigma_{0}^{\prime}$ in the corresponding $\omega_{r}^{i d e m}$ derivation $D$.

Given above observations it is straightforward to construct the mapping function $\alpha$ and the required derivations for both directions of the proof.

\footnotetext{
${ }^{4}$ In this case a finite number of duplicate propagations would also not be a problem.
} 
For multi-headed propagation rules, reapplication is often cheaper than maintaining and checking a history. The experimental results of Section 5 confirm this. Of course, reapplying a body can be arbitrarily expensive. To estimate the cost of reapplication versus the cost of maintaining a history, heuristics can be used.

\subsection{Deriving Idempotence}

The main challenge lies in automatically deriving that a CHR constraint is idempotent. A wide class of idempotent CHR constraints should be covered:

Example 8. Many constraint solvers contain a rule such as:

$$
\text { in }\left(\mathrm{X}, \mathrm{L}_{1}, \mathrm{U}_{1}\right) \backslash \text { in }\left(\mathrm{X}, \mathrm{L}_{2}, \mathrm{U}_{2}\right) \Leftrightarrow \mathrm{L}_{2} \leq \mathrm{L}_{1}, \mathrm{U}_{2} \geq \mathrm{U}_{1} \mid \text { true. }
$$

Here, 'in $(\mathrm{X}, \mathrm{L}, \mathrm{U})$ ' denotes that the variable $\mathrm{X}$ lies in the interval $[\mathrm{L}, \mathrm{U}]$. The in/3 constraint is probably idempotent (it depends on the preceding rules). There is an important difference though with the leq/2 constraint in Example 7: by the time the constraint is told for the second time, the earlier told duplicate may now be replaced with a syntactically different constraint - in this case: a constraint representing a smaller interval domain.

Theorem 4 provides a sufficiently strong syntactic condition for determining the idempotence of a CHR constraint. It uses arbitrary preorders on the constraint's arguments. For the three arguments of the in $/ 3$ constraint in Example 8 for instance, the preorders $=, \leq$ and $\geq$ can be used respectively.

Let $b i(B)$ and $\operatorname{chr}(B)$ denote the conjunction of built-in respectively CHR constraints that occur in a constraint conjunction $B$. Then:

Theorem 4. A CHR constraint type $c / n$ is idempotent in $\mathcal{P}$ under $\omega_{r}^{\text {idem }}$ if for preorders $\triangleleft_{1}, \ldots, \triangleleft_{n}$ :

1. There exists a rule of the form " $c\left(y_{1}, \ldots, y_{n}\right) \backslash c\left(x_{1}, \ldots, x_{n}\right) \Leftrightarrow G \mid$ true." in $\operatorname{HNF}(\mathcal{P})$ with $\mathcal{D}_{\mathcal{H}} \models\left(x_{1} \triangleleft_{1} y_{1} \wedge \ldots \wedge x_{n} \triangleleft_{n} y_{n}\right) \rightarrow G$.

Let $\rho$ be the first such rule occurring in the $H N F(\mathcal{P})$ sequence.

2. All rules in $H N F(\mathcal{P})$ prior to $\rho$ that contain an occurrence of $c / n$ have a trivial body 'true', and do not contain any removed occurrences apart from possibly that $c / n$ occurrence.

Consider a set of $n$ mutually distinct variables $V=\left\{x_{1}, \ldots, x_{n}\right\}$. For all removed occurrences of $c / n$ in $H N F(\mathcal{P})$ that can be renamed to the form

$$
H_{k} \backslash H_{r_{1}}, c\left(x_{1}, \ldots, x_{n}\right), H_{r_{2}} \Leftrightarrow G \mid B
$$

$\left(H_{k}, H_{r_{1}}\right.$, and $H_{r_{2}}$ may be empty), such that $\neg \exists c\left(y_{1}, \ldots, y_{n}\right) \in H_{k} \cup \operatorname{chr}(B)$ : $\mathcal{D}_{\mathcal{H}} \models G \wedge$ bi $(B) \rightarrow\left(x_{1} \triangleleft_{1} y_{1} \wedge \ldots \wedge x_{n} \triangleleft_{n} y_{n}\right)$, define $\Phi=\pi_{V}(G \wedge$ bi $(B))$. For each of these occurrences, either $\mathcal{D}_{\mathcal{H}}=\Phi \leftrightarrow$ false, or conditions 3 and 4 hold:

3. There exists a rule in $H N F(\mathcal{P})$ that can be renamed such that it has form "c $\left(x_{1}, \ldots, x_{n}\right) \Leftrightarrow G \mid B$ ", with bi $(B)=B$ and $\mathcal{D}_{\mathcal{H}}=\Phi \rightarrow(G \wedge B)$.

Let $\rho^{\prime}$ be the first such rule occurring in the $\operatorname{HNF}(\mathcal{P})$ sequence. 


\begin{tabular}{|c|c|c|c|c|c|c|c|c|c|c|}
\hline & \multicolumn{2}{|r|}{ SWI } & \multicolumn{3}{|c|}{ JCHR } & \multirow{2}{*}{$\mid \begin{array}{c}\text { total \# } \\
\text { rules }\end{array}$} & \multicolumn{4}{|c|}{$n$-headed propagation rules } \\
\hline & history & non-react & history & non-react & non-react+ & & $n=1$ & $n=2$ & $n=3$ & $n>3$ \\
\hline FIBBO $(1000)$ & 15,929 & $4,454(28 \%)$ & 70 & $67(95 \%)$ & $21(30 \%)$ & 3 & 1 & - & 1 & - \\
\hline $\operatorname{FIBBO}(3000)$ & timeout & timeout & 542 & $464(85 \%)$ & $153(28 \%)$ & 3 & 1 & - & 1 & - \\
\hline FLOYD-WARSH $(30)$ & 11,631 & $9,706(83 \%)$ & 368 & $188(51 \%)$ & $186(51 \%)$ & 21 & 3 & 2 & 1 & - \\
\hline $\operatorname{INTERPOL}(8)$ & 5,110 & $1,527(30 \%)$ & 43 & $41(95 \%)$ & $37(86 \%)$ & 5 & - & 2 & - & - \\
\hline MANNERS(128) & 849 & $561(66 \%)$ & 328 & $322(98 \%)$ & $317(97 \%)$ & 8 & - & - & 1 & - \\
\hline NSP_GRND(12) & 547 & $169(31 \%)$ & 10 & $6(60 \%)$ & $5(50 \%)$ & 3 & 1 & 1 & - & - \\
\hline NSP_GRND(36) & 81,835 & $10,683(13 \%)$ & 1,434 & $502(35 \%)$ & $494(34 \%)$ & 3 & 1 & 1 & - & - \\
\hline $\operatorname{SUM}(1000,100)$ & 6,773 & $3,488(51 \%)$ & 215 & $135(63 \%)$ & $N / A$ & 4 & - & 1 & - & - \\
\hline TURING(20) & 10,372 & $7,387(71 \%)$ & 761 & $280(37 \%)$ & $276(36 \%)$ & 11 & 1 & 4 & 1 & 5 \\
\hline WFS(200) & 2,489 & $2,143(86 \%)$ & 71 & $67(94 \%) \mid$ & $67(94 \%)$ & 44 & - & 4 & - & - \\
\hline
\end{tabular}

Table 1. Benchmark results (in average milliseconds) for non-reactive CHR rules.

4. All rules in $H N F(\mathcal{P})$ prior to $\rho^{\prime}$ that contain an occurrence of $c / n$ can be renamed to " $H_{k} \backslash H_{r} \Leftrightarrow G \mid B$ " with $H_{k}++H_{r}=H_{1}+\left[c\left(x_{1}, \ldots, x_{n}\right)\right]++H_{2}$, such that either

- $\mathcal{D}_{\mathcal{H}} \models \Phi \rightarrow \neg G$; or

- $H_{r} \subseteq\left[c\left(x_{1}, \ldots, x_{n}\right)\right] \wedge(b i(B)=B) \wedge \mathcal{D}_{\mathcal{H}} \models(\Phi \wedge G) \rightarrow B$; or

- $\exists c\left(y_{1}, \ldots, y_{n}\right) \in H_{1} \cup H_{2}: \mathcal{D}_{\mathcal{H}} \models(\Phi \wedge G) \rightarrow\left(x_{1} \triangleleft_{1} y_{1} \wedge \ldots \wedge x_{n} \triangleleft_{n} y_{n}\right)$.

Proof Sketch. By Definition 9, we have to show that adding a $c / n$ constraint makes no essential changes to the execution state if a duplicate constraint was added earlier in the same derivation. The proof considers two cases: either the duplicate constraint, or a constraint derived from it, is still in the store, or it has been removed. We show that, if the theorem's conditions hold, in both these cases the newly told duplicate is removed, and that it only makes idempotent state changes before that. The complete, formal proof can be found in [11].

\section{Evaluation}

We implemented the optimizations introduced in this paper in the K.U.Leuven CHR system [3,6] for SWI-Prolog, and in the K.U.Leuven JCHR system [4] for Java, and evaluated them using typical CHR benchmarks and constraint solvers $^{5}$. Benchmark timings are given in Tables 1 and 2. The history columns give the reference timings (in milliseconds) when using a propagation history.

The non-react columns in Table 1 contain the results when the optimizations of Section 3 are used. For the non-react+ measurements, loop-invariant code motion was applied to the identifier comparisons (see Section 3.3; currently only implemented in $\mathrm{JCHR}^{6}$ ). Only for the sum benchmark the the history was eliminated using the optimization of Section 3.2 (code motion is of course not applicable $(N / A)$ in this case). Table 2 shows the results for the idempotence-based history elimination of Section 4.

Significant performance gains are measured all optimizations. The selected benchmarks run about two times faster on average, and scale better as well. Even

\footnotetext{
${ }^{5}$ Information on the benchmarks and the platform used is found in [11, Appendix B].

${ }^{6}$ In JCHR, after code motion, identifier comparisons are integrated in the constraint iterators themselves. These iterators moreover exploit the fact that the stored constraints are often sorted on their identifiers. This can further improve performance.
} 


\begin{tabular}{|c|c|c|c|c|c|c|c|c|c|c|c|}
\hline & \multicolumn{2}{|r|}{ SWI } & \multicolumn{2}{|c|}{ JCHR } & & & \multicolumn{2}{|r|}{ SWI } & \multicolumn{2}{|r|}{ JCHR } & \multirow{2}{*}{ \# } \\
\hline & history & idempotence & hist. & idempot. & & & history & idempotence & hist. & idempot. & \\
\hline 21$)$ & 222,622 & 17,611 & 8 & $5(62 \%)$ & |||| $15 / 27$ & EQ( & 3,465 & $931 \quad(56 \%)$ & $\overline{477}$ & 19 & $1 / 4$ \\
\hline 42) & timeout & timeout & 54 & $28(52 \%)$ & $\mid 15 / 27$ & LE & 3,806 & $1,236(32 \%)$ & 85 & $35(41 \%)$ & $1 / 4$ \\
\hline NSP_G & 547 & $164(30 \%)$ & 10 & $6(60 \%)$ & $2 / 3$ & $\operatorname{NSP}(12)$ & 1,454 & $1,036(71 \%)$ & 12 & $8(67 \%)$ & $2 / 3$ \\
\hline NSP_GRND $(36)$ & 81,835 & $10,485(13 \%)$ & 1,365 & $496(36 \%)$ & $2 / 3$ & $\operatorname{NSP}(36)$ & ||timeout & timeout & $\mid 1,434$ & $621(43 \%)$ & $2 / 3$ \\
\hline TIMEPOINT(16) & 1,684 & $1,312(78 \%)$ & 404 & $317(78 \%)$ & $2 / 7$ & $\mid \operatorname{MinMax}(15)$ & 4,826 & $3,631 \quad(75 \%)$ & 133 & $82(61 \%)$ & $\mid 6 / 54$ \\
\hline
\end{tabular}

Table 2. Benchmark results (in average milliseconds) for idempotent propagation rules. The '\#' columns give the number of propagation rules over the total number of rules.

though no numbers are shown, it is moreover clear that the space complexity of the propagation histories has become optimal. Unoptimized, the worst-case space consumption of a propagation history is linear in the number of rule applications (cf. Example 6). Using our optimizations, histories consume no space at all. In extreme cases, this even improves the space complexity of the entire handler.

\section{Conclusions}

Related Work A preliminary version of this paper covering only Section 3.3 of the present paper appeared in [7]. The present paper completes this earlier work by introducing propagation history elimination based on unobservedness and idempotence, and by providing a more extensive experimental evaluation.

Section 3.2 can be seen as an extension and formalization of an optimization briefly presented in [5]. This ad-hoc optimization was restricted to fixed CHR constraints, and lacked a formal correctness proof.

Since the propagation history contributes to significant performance issues when implementing CHR in a tabling environment (see e.g. [12]), [13] proposes an alternative set-based CHR semantics, and argues that it does not need a propagation history. Our results, however, show that abandoning CHR's familiar multiset-based semantics is not necessary: indeed, our optimizations eliminate the history-related performance issues whilst preserving the $\omega_{r}$-semantics.

Conclusions Whilst there is a vast research literature on CHR compilation and optimization, propagation histories never received much attention. Maintaining a propagation history, however, comes at a considerable runtime cost, both in time and in space. In this work, we resolved this discrepancy by introducing several innovative optimization techniques that circumvent the maintenance of a history for the majority of CHR propagation rules:

- For non-reactive CHR propagation rules, we showed that very cheap constraint identifier comparisons can be used. These comparisons can moreover be moved early in the generated nested iterations, thus pruning the search space of possible partner constraints. We also formally identified the class of non-reactive rules for which the history can simply be eliminated.

- Whilst rules in general-purpose CHR programs are mostly non-reactive, CHR handlers that specify a constraint solver are typically highly reactive. We therefore introduced the concept of idempotence, and found that most rules in the latter handlers are idempotent. We showed that if a propagation 
rule is idempotent, the rule may safely be applied more than once matching the same combination of constraints. Interestingly, reapplication is mostly cheaper than maintaining and checking a history. We also presented a sufficient syntactic condition for the idempotence of a CHR constraint.

We proved the correctness of all our optimizations and analyses in the formal framework of CHR's refined operational semantics [8], and implemented them in two state-of-the-art CHR systems [3,4]. Our experimental results show significant performance gains for all benchmarks containing propagation rules.

Acknowledgments The author thanks Tom Schrijvers for his invaluable aid in the implementation of the optimizations in the K.U.Leuven CHR system. Thanks also to Bart Demoen and the anonymous referees of CHR 2008 and ICLP 2008 for their useful comments on earlier versions of this paper.

\section{References}

1. Frühwirth, T.: Theory and practice of Constraint Handling Rules. J. Logic Programming, Special Issue on Constraint Logic Programming 37(1-3) (1998) 95-138

2. Sneyers, J., Van Weert, P., Schrijvers, T., De Koninck, L.: As time goes by: Constraint Handling Rules - A survey of CHR research between 1998 and 2007. Submitted to Journal of Theory and Practice of Logic Programming (2008)

3. Schrijvers, T., Demoen, B.: The K.U.Leuven CHR system: Implementation and application. In: CHR 2004: Selected Contributions, Ulm, Germany (2004) 8-12

4. Van Weert, P., Schrijvers, T., Demoen, B.: K.U.Leuven JCHR: a user-friendly, flexible and efficient CHR system for Java. In: CHR 2005: Proc. 2nd Workshop on Constraint Handling Rules, Sitges, Spain (2005) 47-62

5. Duck, G.J.: Compilation of Constraint Handling Rules. PhD thesis, University of Melbourne, Australia (December 2005)

6. Schrijvers, T.: Analyses, optimizations and extensions of Constraint Handling Rules. PhD thesis, K.U.Leuven, Belgium (June 2005)

7. Van Weert, P.: A tale of histories. In: CHR 2008: Proc. 5th Workshop on Constraint Handling Rules, Hagenberg, Austria (2008) 79-94

8. Duck, G.J., Stuckey, P.J., García de la Banda, M., Holzbaur, C.: The refined operational semantics of Constraint Handling Rules. [14] 90-104

9. Schrijvers, T., Stuckey, P.J., Duck, G.J.: Abstract interpretation for Constraint Handling Rules. In Barahona, P., Felty, A., eds.: PPDP '05: Proc. 7th Intl. Conf. Princ. Pract. Declarative Programming, Lisbon, Portugal, ACM (2005) 218-229

10. Schrijvers, T., Demoen, B.: Antimonotony-based delay avoidance for CHR. Technical Report CW 385, K.U.Leuven, Dept. Computer Science (July 2004)

11. Van Weert, P.: Optimization of CHR propagation rules: Extended report. Technical Report CW 519, K.U.Leuven, Dept. Computer Science (August 2008)

12. Schrijvers, T., Warren, D.S.: Constraint Handling Rules and tabled execution. [14] 120-136

13. Sarna-Starosta, B., Ramakrishnan, C.: Compiling Constraint Handling Rules for efficient tabled evaluation. In Hanus, M., ed.: PADL 2007. Volume 4354 of LNCS., Nice, France, Springer (January 2007) 170-184

14. Demoen, B., Lifschitz, V., eds.: ICLP 2004. Volume 3132 of LNCS. Springer, Saint-Malo, France (September 2004) 